\title{
ASSESSMENT OF ULTRACAMD PERFORMANCE IN AN ARID ENVIRONMENT - CASE STUDY IN LIBYA
}

\author{
Abdulhamed S. Gneeniss, Jon P. Mills, Pauline E. Miller \\ School of Civil Engineering and Geosciences, Newcastle University, UK (a.s.gneeniss, j.p.mills, p.e.miller)@ncl.ac.uk
}

KEY WORDS: Large format cameras, Bundle adjustment, Self-calibration, Arid Environment, Systematic errors

\begin{abstract}
:
Large format digital aerial cameras are now in widespread commercial operation. Despite the advantages of the new cameras over their traditional film counterparts, systematic image errors have been observed in all existing large format digital cameras. Organizations such as the USGS and EuroSDR have therefore focused efforts on calibration, validation and certification of digital camera systems. However, to-date, few studies have assessed the effects of extreme temperature and humidity variations on these camera systems, and the potential for self-calibration in this respect. This research addresses these issues through investigation of the UltraCamD, with test data acquired over a range of climatic zones in Libya. This presentation will report the preliminary results of self-calibration using a bundle block adjustment for an UltraCamD system, based upon aerial data acquired for two test sites during a single field campaign. The datasets were flown at two different flying heights and incorporate differing block geometries. A SOCET SET (v5.4.1) digital photogrammetric workstation was used to triangulate the imagery with investigation of different tie point densities. Following this, a self-calibrating bundle block adjustment was performed using the BLUH software provided by the University of Hannover. Initial testing investigated the influence of tie point density and different coordinate systems and datums on the bundle adjustment results. Further testing was then performed within BLUH in order to determine the optimal set of additional parameters, compensating for systematic image errors and impact upon object space coordinates of independent check points and ground control points.
\end{abstract}

\section{INTRODUCTION}

Due to the apparent advantages of digital cameras over conventional film cameras in geometry and image quality, digital cameras are now in widespread commercial use. As with aerial film cameras, the manufacturer's laboratory calibration is still being applied to commercially operational digital cameras. Despite the precision and accuracy of the measurements provided by laboratory calibration, practical tests have shown that without self calibration, systematic image errors are clear for all existing large format digital cameras (Alamus, 2006; Honkavarra, 2006; Kruck, 2005; Passini et al., 2008; Scholz et al., 2009). It has also been reported in the literature that the reasons for these errors are due to the differences between the laboratory and the actual mission environment, atmospheric refraction (Tachibana et al., 2008), multi-head integration (Dorsel et al., 2007) and changes in the operating temperature (Yastikli, 2004). Systematic errors which represent the difference between the mathematical model of the perspective geometry and the real image geometry can be detected and respected by self-calibration with additional parameters. Different sets of additional parameters have been developed to determine and compensate for these systematic errors through self-calibration. Examples include the Ebner 12 and Grün 44 additional parameters (Passini et al, 2008). The accuracy of the obtained additional system parameters demands a strong geometry in the block and accurate ground control points in the test field (Honkavaara et al., 2008). Due to the differences in design and working concepts of current digital aerial systems, new special additional parameters have been designed to account for the specific geometry of each camera model. These new additional parameters have been introduced into the Hannover bundle block adjustment program BLUH (Büyüksalih et al, 2006). However, practical tests have shown that even the special additional parameters which are designed to account for the specific geometry of the new digital large format cameras sometimes have limited effects and have not been found to improve accuracy at independent check points. (Baz et al, 2006; Büyüksalih et al, 2006; Jacobsen, 2007)

The aim of this research is to investigate the effects of extreme environmental conditions on a digital airborne UltraCamD camera system across Libya, and investigate the suitability of existing camera calibration methods to compensate for any identified systematic errors.

\section{METHODOLOGY}

The initial camera calibration tests were based on two datasets of real world mapping missions acquired using an UltraCamD camera model owned by the Libyan Centre for Remote Sensing and Space Science (LCRSSS). These datasets were flown at two different flying heights with differing block geometry and located in areas which have different climatic conditions.

The first mission was flown over the Sidi-Assaid area located approximately 67 kilometres south-east of Tripoli on January $29^{\text {th }}, 2007$. The second took place over the Al Gazal area (northeast of Libya) on November $8^{\text {th }}, 2008$. For the Sidi-Assaid area three parallel strips were flown at $1450 \mathrm{~m}$ in a north-south direction with 11 images in each strip. Another two cross strips were flown in an east-west direction with 8 images for each strip, the number of images totalling 49. For the Algazal area four parallel strips were flown at $5650 \mathrm{~m}$ in east-west direction with 18 images in each strip and there were no cross strips in this dataset.

GPS/IMU data for the two test areas were provided along with the image datasets. For Sidi-Assaid area the data was provided with $\pm 4 \mathrm{~cm}$ accuracy in horizontal and $\pm 9 \mathrm{~cm}$ in vertical. For the $\mathrm{Al}$ Gazal area, the accuracy of the GPS/IMU data was $\pm 9 \mathrm{~cm}$ in horizontal and $\pm 14 \mathrm{~cm}$ in vertical. Different ground control point (GCP) configurations were available for the two areas. In Sidi-Assaid 22 well distributed cultural features points were 
surveyed using static GPS methods with 20 minutes observation time. For the Algazal area 9 premarked ground control points were surveyed through a similar approach. In both cases, the GPS data was post-processed in Leica Geo-Office.

\subsection{Experimental Strategy}

As only 9 ground control points were available for the Al Gazal dataset, in the Sidi-Assaid area the priority was given to use at least 9 well distributed ground control points in the bundle adjustment and use the remaining points as independent check points. The tests focused on:

- Investigating the UltraCamD camera geometry using a bundle block adjustment with self-calibration;

- Applying camera self-calibration with different sets of additional parameters using the BLUH bundle adjustment software provide by Hannover University;

- Investigating the effect of tie point density on the bundle adjustment;

- Assessing the influence of different coordinate systems.

\subsection{Data processing}

First, the image measurements for aerial triangulation were performed using the automatic tie point measurements model, APM, within the SOCET SET software (v 5.4.1). All points were stereoscopically re-measured to make sure they were properly located. Ground control points were identified and manually and stereoscopically measured. For the two test areas, two sets of tie point densities were prepared, (300/1000 for SidiAsaid and 400/1000 for Al Gazal area). In this study all measurements were exported into BLUH format which is bundle adjustment software developed and provided by the University of Hannover.

The influence of map projections on the bundle adjustment was also considered. As stated by Jacobsen (2010), "In theory, the best coordinate system is a tangential plane system to the earth ellipsoid". Therefore, to eliminate the effect of earth curvature, which will mainly cause deformation in the vertical component, it was decided to use orthogonal tangential coordinates for the two test areas. This also allowed direct comparison of results obtained from areas located in different regions. Transformation of ground control points from GPS coordinates into tangential coordinates was made using the BLUH BLTRA module.

Bundle adjustment was performed within BLUH as follows: Input data from different files for the different sites and tie points densities were imported individually using the BLPRE module. This allowed the data to be converted into BLUH format. Focal length was automatically identified from the input data files, whereas the principal point coordinates were defined and inserted manually during BLPRE module setup.

The BLOR module was used to define the block geometry approximate orientations of the images. This configuration was defined by ground control points. At this stage data snooping was used to detect any Blunders. Also input images were grouped into strips and unique camera number for each strip to correct for any shifts and drifts in the kinematic GPS data in the bundle adjustment. The input parameters for the bundle block adjustment were prepared by the module BLIM. In this study, the investigation of camera performance and self-calibration was performed using different sets of additional parameters. In the first run no self-calibration was made and the robust estimator option was introduced to detect and eliminate any blunders from the input data. In the second run the 1-12 BLUH standard set of additional parameters for usual perspective was used. These parameters are mainly designed to fit general image deformations such as affinity, tangential distortion, radial symmetric distortion and general deformation. The parameters from 13-20 were used, this set includes correction for focal length and principal point coordinates. Also determination and compensation of GPS shift drift and offset. The final run included special additional parameters $42-73$ for UltraCamD. These parameters are intended to respect any deformation in the sub-images geometry.

The BLUH bundle block adjustment module is based on the colinearity condition equations. Observations for the adjustment are photo coordinates, control point coordinates and coordinates of the projection centers. The camera interior orientation can be improved by introducing the additional parameters when the original camera parameters are already known before the adjustment. The other unknowns are exterior orientations and additional parameters in the case when self-calibration will be applied.

\section{RESULTS AND DISCUSSION}

Testing was carried out to assess the optimum tie point configuration. Table 1 shows the minimum and maximum number of tie points per image in each block. This highlights that use of 300-400 tie points does not provide enough redundancy as the number of points will not be sufficient to precisely estimate any deformation in the aerial images. This is particularly relevant in the case of the UltraCamD where the large virtual image is constructed by combining 9 smaller images. Therefore, it was decided to perform self-calibration using t the 1000 tie points option, with the tangential coordinate system.

\begin{tabular}{|c|c|c|}
\hline & \multicolumn{2}{|c|}{ Number of points per-image } \\
\cline { 2 - 3 } & Minimum & Maximum \\
\hline $\begin{array}{c}\text { Sidi-Assaid } \\
300 \text { tie pts }\end{array}$ & 22 & 37 \\
\hline $\begin{array}{c}\text { Sidi-Assaid } \\
1000 \text { tie pts }\end{array}$ & 91 & 137 \\
\hline $\begin{array}{c}\text { Al Gazal } \\
300 \text { tie pts }\end{array}$ & 9 & 33 \\
\hline $\begin{array}{c}\text { Al Gazal } \\
1000 \text { tie pts }\end{array}$ & 34 & 71 \\
\hline
\end{tabular}

Table 1 Minimum and maximum number of tie points per image in each block.

In accordance with the methodology described in Section 2, the bundle adjustment was performed for the two sites using the different sets of additional parameters. Figures 1 and 2 show the obtained accuracy from the two sites, it is obvious that after introducing the additional parameters, BLUH 12AP and 20AP, to the bundle adjustment the accuracy of object points increased significantly. Even after introducing additional parameters $42-$ 73 into the bundle adjustment (which are specially designed to account for the geometry of the UltraCamD) the accuracy did not improve as expected. Indeed it actually deteriorated in the $\mathrm{Z}$ axis.

For the Sidi-Assaid area the expected accuracy which is in the range of 0.5 pixel in horizontal and one pixel in vertical nearly matches the estimated accuracy from the bundle adjustment. For the Al Gazal area, the estimated accuracy was close to the 
expected accuracy in horizontal. However, the difference was quite large in vertical accuracy.

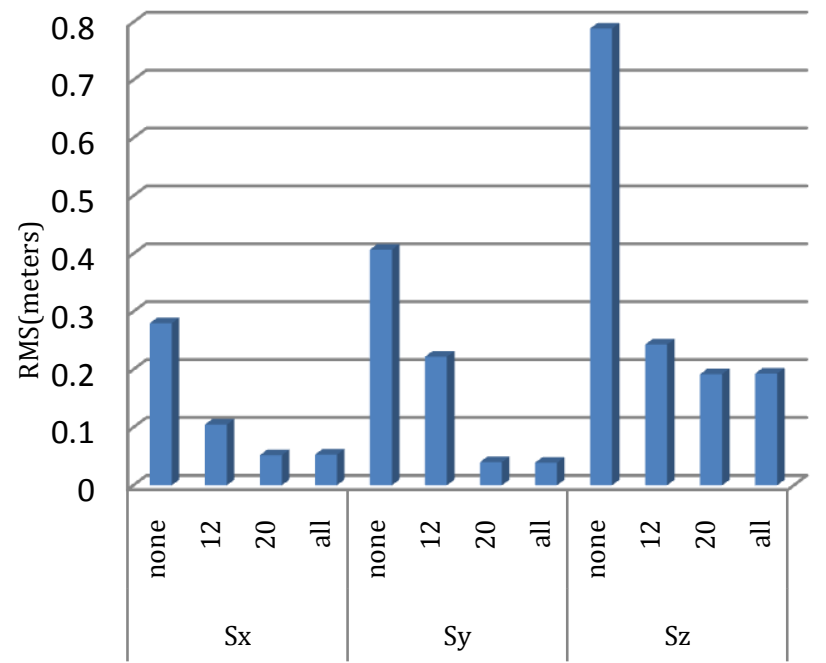

Figure 1 Accuracy at independent check points for SidiAssaid test site.

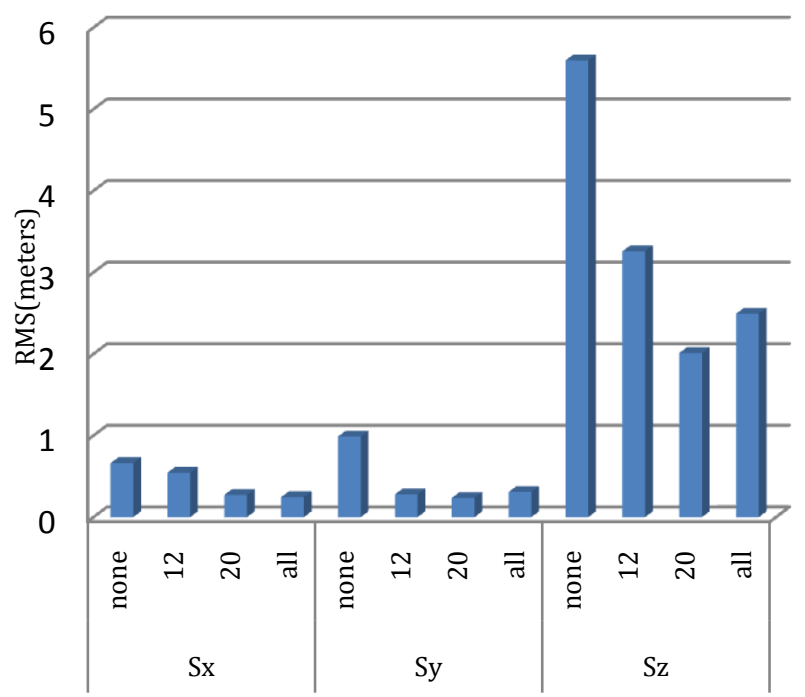

Figure 2 Accuracy at ground control points for Al Gazal area.

A direct comparison of results for the two datasets is shown in Figure 3. For both datasets, the horizontal accuracy is in the range of 0.5 pixels after introducing the additional parameters in the bundle adjustment. This improvement was observed after introducing the 12 additional parameters to compensate for general and radial distortions. Also the accuracy was even better after using the additional parameters from 1 to 20 which included other parameters to compensate for any errors in the GPS data in terms of shifts and drifts. However larger variation was observed in the vertical accuracy, with the Al Gazal dataset notably poorer than the Sidi-Assaid results in all cases. This could be due to radiometric degradation and artefacts which were observed in the input dataset. Another factor which may also contribute to this low accuracy is that the datasets were supplied by the LCRSSS (Libyan Centre for Remote Sensing and Space Science) following processing using an old version of the UltraCamD software. In the latest version, the camera manufacturer has improved the methods of image merging and stitching in order to reduce systematic image errors (Jacobsen, 2007).

Pixels

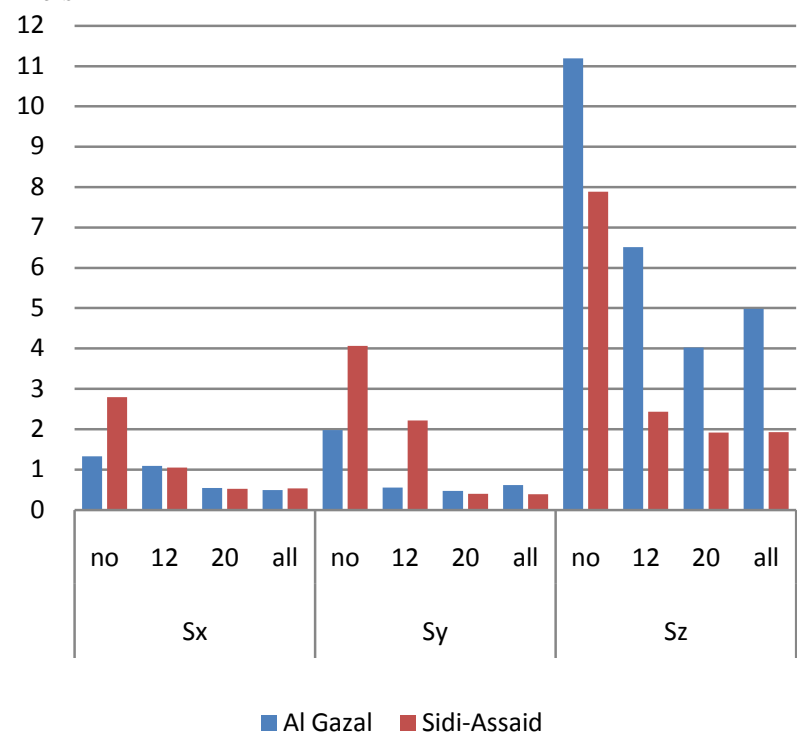

Figure 3 Accuracy comparison (pixels) between Sidi-Assaid and $\mathrm{Al} \mathrm{Gazal} \mathrm{areas.}$

Figures 4 and 5 show the averaged image residuals of the two test areas for the case of no additional parameters, 12 additional parameters, and all additional parameters (including the UltraCamD specific parameters) which indicate large systematic errors. By comparing the residuals obtained when no self calibration was made, the images residuals tend to have a similar pattern, particularly in the upper part.

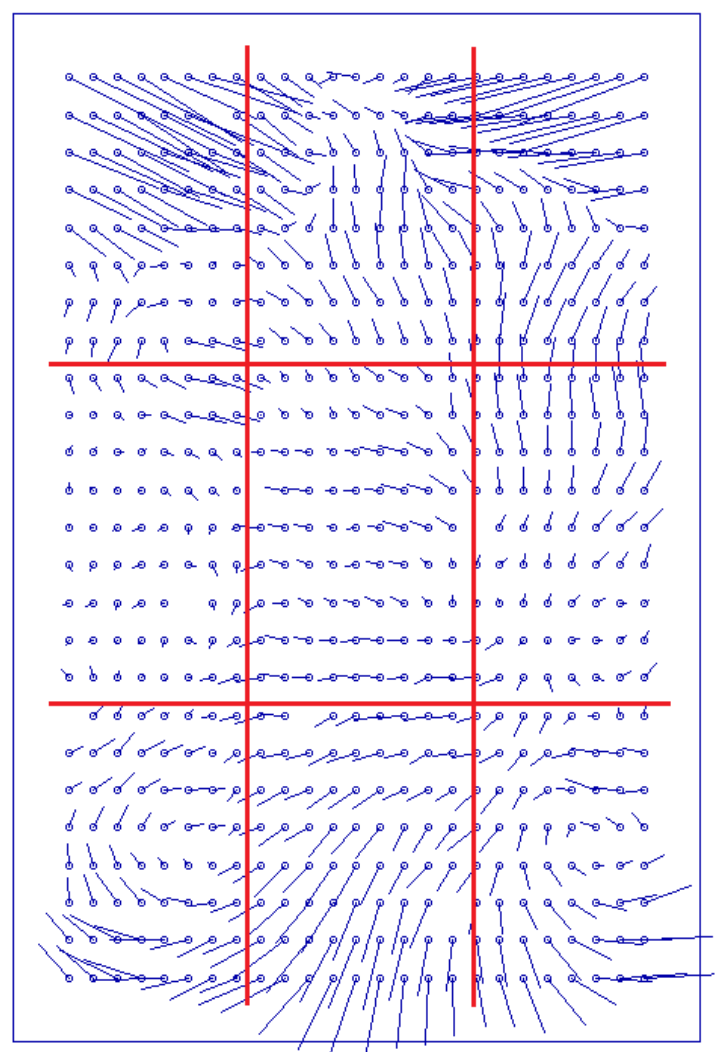

a-No-self calibration $\longmapsto 9.0$ 

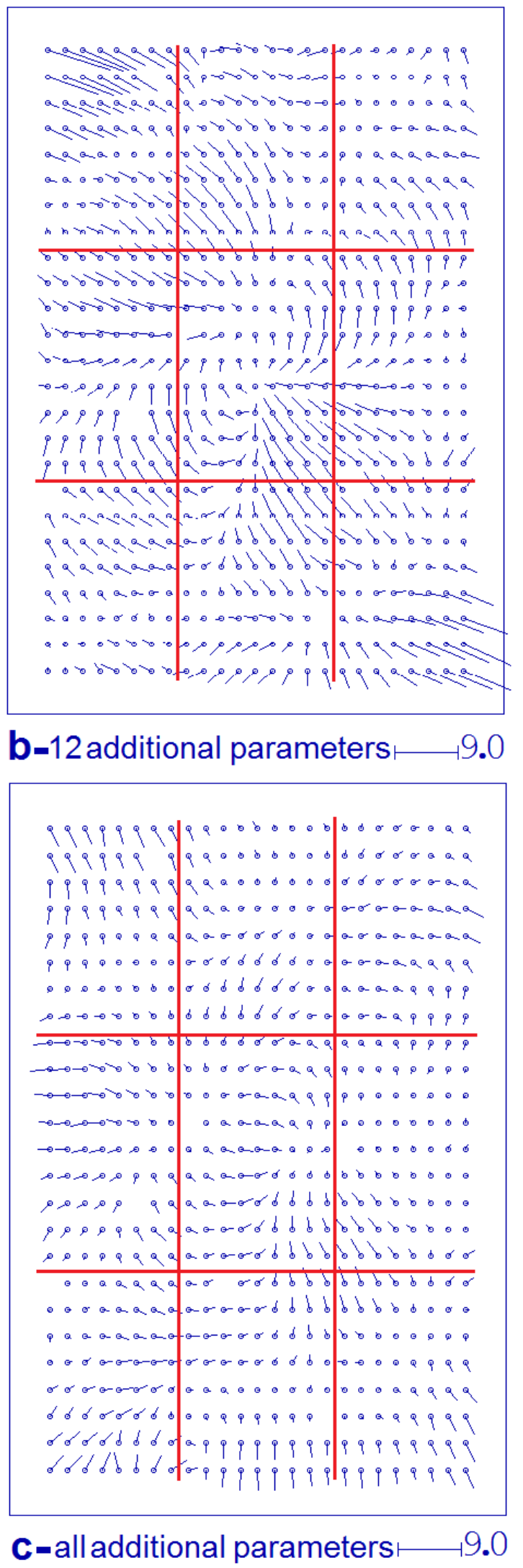

Figure 4 (a, b, c) Averaged image residual for Sidi-Assaid area.

Moreover, these figures show that most of the systematic image errors can be reduced with the 12 general additional parameters.
The factor which could contribute to these large systematic errors is that the aerial imagery was post processed using the standard stitching algorithm using the old version of the UltraCamD software. Also by comparing these findings to other results presented by Ladstädter et al (2010) that the camera factory calibration maybe lost and the calibration data was no longer valid in the camera post processing stage, which means the camera needs to be recalibrated.
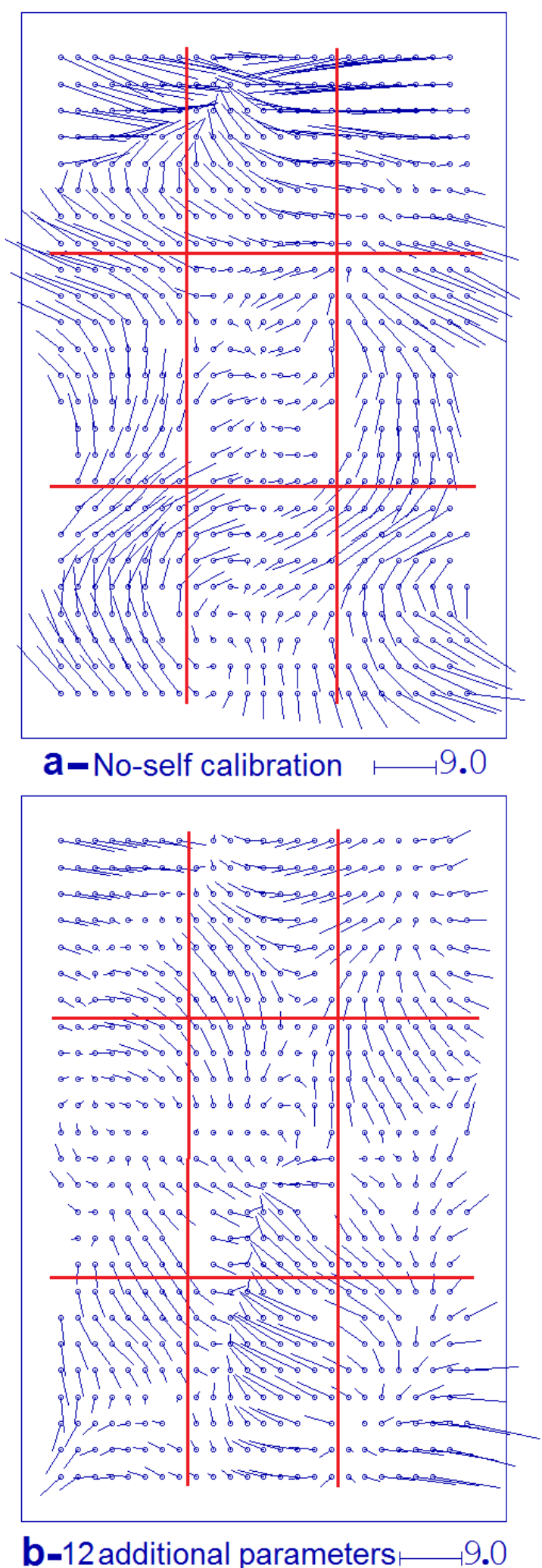

b-12 additional parameters 


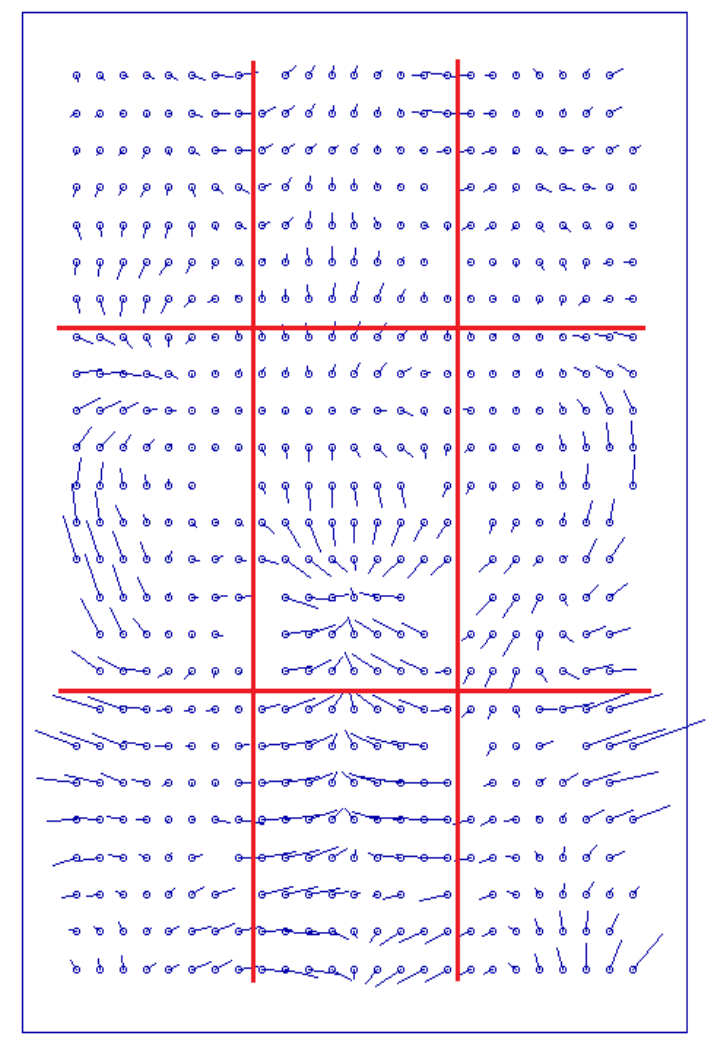

\section{C-all additional parameters $\longmapsto 9.0$}

Figure $5(\mathrm{a}, \mathrm{b}, \mathrm{c})$ Averaged image residual for Al Gazal area.

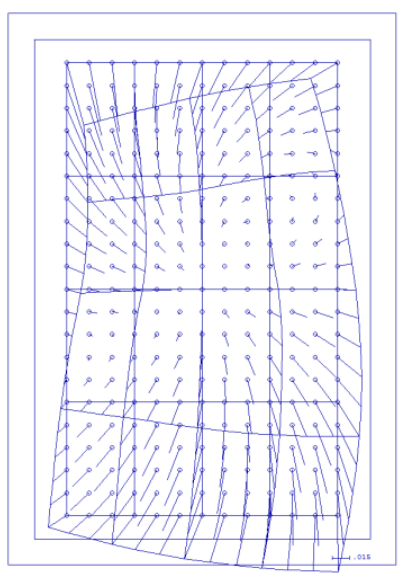

12 additional parameters

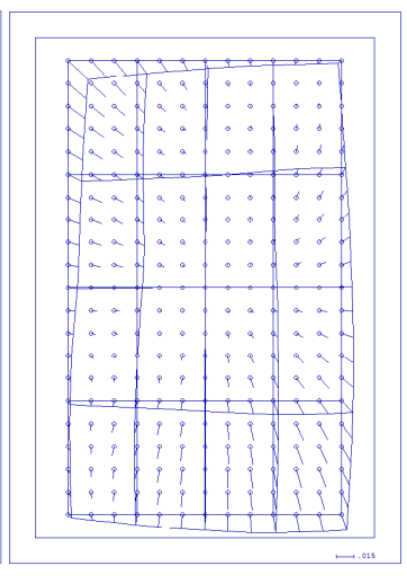

All additional parameters
Figure 6 Effect of additional parameters on the image space for the Sidi-Assaid test area.

Figures 6 and 7 highlight the effect of additional parameters on the image space for Sidi-Assaid and Algazal areas. These results also showed that most systematic image errors can be fitted with the standard additional parameters (1-12) of the BLUH software.

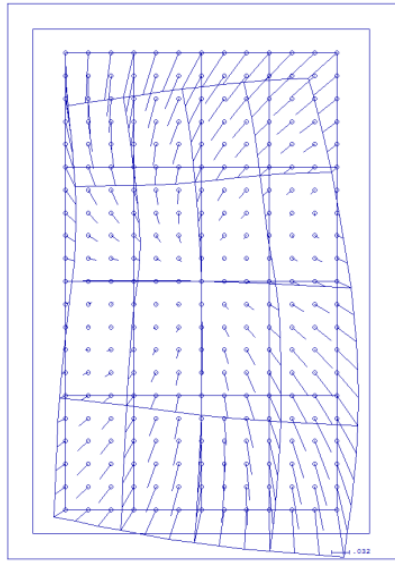

12 additional parameters

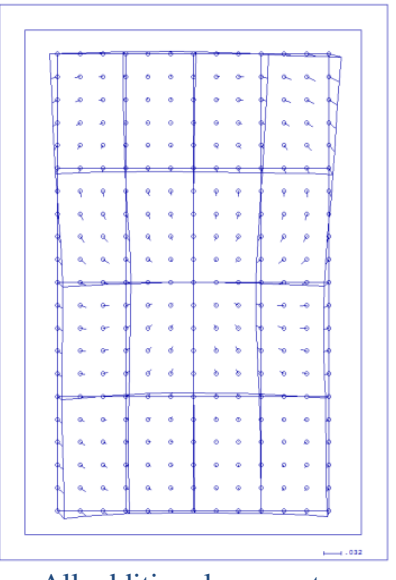

All additional parameters
Figure 7 Effect of additional parameters on the image space for the $\mathrm{Al} \mathrm{Gazal} \mathrm{test} \mathrm{area.}$

In both cases the special UltraCamD additional parameters have only a limited influence on the adjustment. Furthermore, these special Additional Parameters have sometimes deteriorated the obtained accuracy as evidenced in Figure 2 which means that they are unable to fit the systematic effects in these cases.

\section{CONCLUSION}

The datasets used in these tests were selected from real world mapping missions. These preliminary results are specific to the UltraCamD camera model v015 owned by the LCRSSS which has shown large systematic errors compared to previously obtained results from other cameras of the same model. Nevertheless, the results presented here have shown the high potential for self-calibration. The results from the two test areas showed that without self-calibration the achieved horizontal and vertical accuracies were far beyond the expected and acceptable range, thus confirming that the use of self-calibration with additional parameters is necessary.

Accuracy was improved using the BLUH 12 additional parameters and was further improved with the use of 20 additional parameters. In all cases the special UltraCamD additional parameters have displayed only a limited influence on the adjustment. In some cases they were found to degrade the obtained height accuracy, indicating that they are unable to model the remaining systematic errors.

The next phase is to validate these findings using specially designed block structures, different flying heights and additional ground control points. This will allow self-calibration and estimation of any systematic errors with a high degree of precision.

\section{References}

Alamús, R., Kornus, W. and Talaya, J., 2006. Studies on DMC Geometry. ISPRS Journal of Photogrammetry and Remote Sensing, 60, pp. 375 - 386.

Baz, I., Jacobsen, K. and Büyüksalih, G., 2006. Analysis of a bundle block adjustment with UltraCamD images over Istanbul, Turkish-German Geodetic Days, Berlin.

Büyüksalih, G. and Jacobsen, K., 2006. Bundle Block Adjustment with UltraCamD Images. International Archives of 
Photogrammetry, Remote Sensing and Spatial Information Sciences, 36(1), pp. 17-22.

Dorstel, C., 2007. DMC - (R)evolution on Geometric Accuracy. Photogrammetric Week 2007, Wichmann Verlag, pp. 81-88.

Honkavaara, E., Ahokas, E., Hyyppä, J., Jaakkola, J., Kaartinen, H., Kuittinen, R., Markelin, L., Nurminen, K., 2006. Geometric test field calibration of digital photogrammetric sensors. ISPRS Journal of Photogrammetry and Remote Sensing, 60, pp. 387399.

Honkavaara, E., Markelin, L., Ahokas, E., Kuittinen, R., Peltoniemi, J., 2008. Calibrating Digital photogrammetric airborne imaging systems in a test field. ISPRS Journal of Photogrammetry and Remote Sensing, 37, pp 555-560.

Jacobsen, K., 2007. Geometry of Digital Frame Cameras. Proceedings of the ASPRS 2007 annual conference, Tampa, Florida, USA.

Jacobsen, K., 2010. BLUH user manual. University of Hannover, Hannover, Germany.

Kruck, E. J., 2005. Simultaneous Calibration of Digital Aerial Survey Cameras. ISPRS Hannover workshop: High-Resolution Earth Imaging for Geospatial Information, 36(P1), pp. 7.

Passini, R. and Jacobsen, K., 2008. Geometric analysis on digital photogrammetric cameras. ASPRS 2008 Annual Convention, Portland, Oregon.

Scholz, S. and Gruber, M., 2009. Radiometric and Geometric quality aspects of the large format aerial camera UltraCamXp. Proceedings of the Hannover workshop 2009 on HighResolution Earth Imaging for Geospatial Information, 38, pp. 143-147.

Tachibana, K., M. Gruber, H. Shimamura., 2008. Geometric Accuracy investigation of Vexcel UltraCamD. ISPRS Journal of Photogrammetry and Remote Sensing, 37, pp. 611-616.

Yastikli, N., 2004. The effect of system calibration on direct sensor orientation. International Archives of Photogrammetry and Remote Sensing, 35(B1), pp. 205-209.

\section{Acknowledgements}

The authors are grateful to the Libyan Centre for Remote Sensing and Space Science for providing the image data and all people involved in data acquisition, processing and field survey. The authors would also like to thank Dr. K. Jacobsen from Hannover University for the use of BLUH bundle adjustment software. 